\title{
MINERALOGIA DE PARTÍCULAS ENVOLVIDAS NA FORMAÇÃO DE GRADIENTE TEXTURAL EM UM ARGISSOLO SUBTROPICAL ${ }^{(1)}$
}

\author{
Edson Campanhola Bortoluzzi ${ }^{(2)}$, Miguel Pernes ${ }^{(3)} \&$ Daniel Tessier ${ }^{(3)}$
}

\begin{abstract}
RESUMO
A presença de gradiente textural é comum e marcante em Argissolos. O objetivo do trabalho foi estudar a mineralogia das partículas envolvidas no processo de formação de gradiente textural em um solo subtropical do Rio Grande do Sul. Amostras de um Argissolo Vermelho-Amarelo distrófico abrúptico, material parental sedimentar, foram coletadas em duas trincheiras localizadas em distintas posições no relevo da área do departamento de solos da Universidade Federal de Santa Maria. No perfil 1, sob campo nativo e posicionado na meia encosta de uma colina com $9 \%$ declividade, amostras foram coletadas nos horizontes $A_{1}, A_{2}, A B e$ Bt. No perfil 2, sob vegetação espontânea e localizado no topo plano da colina, amostras foram coletadas nos horizontes $\mathrm{A}_{1}, \mathrm{~A}_{2}, \mathrm{E}$ e Bt. Atributos químicos foram avaliados sobre a fração de solo menor que $2 \mathrm{~mm}$. Amostras de argila dispersa em $\mathrm{NaOH}$, após serem extraídas, foram submetidas à difração laser, discriminando a distribuição do tamanho de partícula envolvida no processo. A fração menor que $0,2 \mu \mathrm{m}$ foi extraída, saturada com $\mathrm{Ca}^{2+} \mathrm{e}$ submetida à difração de raios $\mathrm{X}$ sob os seguintes tratamentos: normal, à temperatura ambiente $(\mathrm{N})$; depois de saturada com etilenoglicol (EG), depois de aquecida a 300 e $550{ }^{\circ} \mathrm{C}$. Os difratogramas de raios X $(\mathrm{N})$ foram submetidos à modelagem matemática com DecompRX. Ambos os perfis apresentaram gradiente textural. Os teores de argila no horizonte $\mathrm{Bt}$ foram 2,9 e 4,4 vezes maiores no perfil 1 e 2 que nos seus respectivos horizontes mais arenosos. Os diagramas de difração laser mostraram enriquecimento no horizonte Bt em partículas de tamanho modal de 0,09 e $0,2 \mu \mathrm{m}$. A mineralogia dessa fração apresentou incremento nos horizontes Bt de interestratificados caulinita-esmectita (C-E) em detrimento das fases vermiculita hidróxi-Al entrecamada (VHE) e ilita-esmectita (I-E). Dessa forma, infere-se que o gradiente textural observado no solo está associado ao processo de migração de C-E com partículas de tamanho modal de 0,09 e 0,2 $\mu \mathrm{m}$. Entretanto, o perfil 2 apresenta
\end{abstract}

\footnotetext{
(1) Recebido para publicação em março de 2007 e aprovado em fevereiro de 2008.

(2) Engenheiro-Agrônomo, Doutor em Ciência do Solo, Professor-Pesquisador Adjunto I da Faculdade de Agronomia e Medicina Veterinária, Universidade de Passo Fundo - FAMV/UPF. Caixa Postal 611, CEP 99001-970 Passo Fundo (RS). E-mail: edsonb@upf.br

(3) Pesquisador do INRA/PESSAC Unité de Physico-chimie et Ecotoxicologie des Sols d'Agrosystèmes Contaminés, Route de Saint Cyr, Versailles, France. E-mail: daniel.tessier@wanadoo.fr
} 
indícios de descontinuidade litológica, indicando que o processo de migração de partículas não é o único responsável pela formação de gradiente textural.

Termos de indexação: interestratificado caulinita-esmectita, translocação de argila, difração laser, ferrólise, bioturbação.

\title{
SUMMARY: MINERALOGY OF PARTICLES INVOLVED IN FORMATION OF A TEXTURE GRADIENT INA SUBTROPICAL ACRISOL
}

\begin{abstract}
Texture gradient is a prominent and ubiquitoud feature of Acrisol. The objective of this paper was to study the particle size distribution and mineralogy involved in the formation of the texture gradient of a subtropical soil in Rio Grande do Sul. Soil samples of a Red-Yellow Acrisol, formed on sedimentary parent material, were collected from trenches at two relief positions in an area of the Universidade Federal de Santa Maria, RS, Brazil. Soil samples were collected from profile 1, located on a slope and under natural pasture, in the horizons $A_{1}, A_{2}, A B$, and $B t$ and from profile 2 , on a hilltop and under spontaneous vegetation, samples were collected in the horizons $A_{1}, A_{2}, E$, and Bt. Chemical attributes of the $<2 \mu \mathrm{m}$ fraction were evaluated. The clay fraction $(<2 \mu \mathrm{m})$, after $\mathrm{NaOH}$ dispersion, was laser-diffracted, to discriminate the size distribution of the particles involved in the process. The $<0.2 \mu \mathrm{m}$ fraction was separated by centrifugation, $\mathrm{Ca}^{2+}$ saturated and $\mathrm{X}$ ray diffracted after the following treatments: control, air-drying at room temperature $(N)$; ethylene-glycol saturation (EG); and heating to 300 and $550{ }^{\circ} \mathrm{C}$. The raw $X$ ray diffractograms were modelled using DecompRX. A texture gradient was observed in both soil profiles. Clay contents in the Bt horizons of profile 1 and 2 were 2.9 and 4.4 times higher than in the surface horizons. Laser diffraction diagrams of the Bt horizon revealed an enrichment in two modal diameter sizes $(0.09$ and $0.2 \mu \mathrm{m})$. The mineralogy of Bt horizons was composed of high kaolinitesmectite (K-S) amounts in detriment of Al-hydroxy interlayered vermiculite (HIV) and illite-smectite (I-S) clay minerals. It is suggested that the textural gradient in the soil profiles was due to migration of K-S particles with a modal diameter of $0.09 \mathrm{im}$ and $0.2 \mu \mathrm{m}$. However, there are pieces of evidence pointing out for lithological discontinuity in profile 2 , indicating that particle migration was not the only process of texture gradient formation.

Index terms: interstratified kaolinite-smectite, clay translocation, laser diffraction, ferrolysis, and bioturbation.
\end{abstract}

\section{INTRODUÇÃO}

O solo como um sistema aberto permite a troca de matéria e energia entre seus horizontes e os ecossistemas adjacentes. A magnitude e a taxa dessas trocas definem a pedogênese e, por conseqüência, as propriedades físico-químicas dos constituintes e a função do solo no contexto ambiental (Wilson, 1999; Brady \& Weil, 2002).

A mudança textural abrupta no perfil de solo vem sendo utilizada como característica diagnóstica para a classificação de solos (Duchaufour, 1997; Soil Survey Staff, 1999; Embrapa, 2006; FAO, 2006), assim como característica interpretativa ligada ao uso e ao manejo de solo e à análise de risco na transferência de contaminantes (Elimelech \& Ryan, 2002; Léguedois et al., 2004). Sua origem no perfil de solo pode estar ligada à herança do material parental, à descontinuidade litológica, decorrente da deposição de camadas sedimentares sobre um perfil já existente (Schaetzl, 1998), e à translocação quando há migração de material no perfil (Phillips, 2007). Nesse último, a mobilidade das partículas finas desencadeia o empobrecimento destas nos horizontes superficiais ao mesmo tempo que há o enriquecimento dos subsuperficiais, processo chamado, respectivamente, de eluviação-iluviação de argila (Soil Survey Staff, 1999). Entretanto, processos secundários, tais como: ferrólise, erosão seletiva, neoformação e remoção também podem contribuir para a formação de gradiente textural (Phillips, 2004, 2007).

Em suma, o processo de ferrólise, freqüente em solos sujeitos aos ciclos de umedecimento e secagem, logo, de redução e oxidação do íon Fe, pode causar o gradiente textural (Almeida et al., 1997; Mafra et al., 2001). Isso porque, na fase de oxidação, passagem de $\mathrm{Fe}^{2+}$ a $\mathrm{Fe}^{3+}$, há liberação de $\mathrm{H}^{+}$capaz de deslocar cátions da estrutura dos argilominerais, destruindoos, o que gera, em estádios avançados, um gradiente textural no perfil (van Ranst \& De Coninck, 2002). A dissolução de argilas finas pelo intemperismo torna o perfil de solo predominantemente mais arenoso no topo. Em outro processo, a erosão seletiva manifestase através do carreamento preferencial de partículas 
finas em razão do fluxo superficial de água e em detrimento das partículas grosseiras. Já, nos horizontes subsuperficiais, o processo de neoformação de argila pode ocorrer em certas condições (Léguedois et al., 2004). Em ambientes de redução, a dissolução e o transporte de $\mathrm{Fe}^{2+}$, como também de outros cátions, para horizontes mais profundos constituem processo preferencial de enriquecimento de materiais em subsuperfície (Wilson, 1999; Brady \& Weil, 2002), interferindo na manutenção, crescimento e neoformação de argilominerais (Pedro, 1979).

Apesar de esses processos agirem concomitantemente, porém com taxas diferentes, o mais comum e responsável predominante pela formação de gradiente textural é a translocação de argila ou iluviaçãoeluviação (Phillips, 2004, 2007). Ocorre principalmente em solos costeiros ou originados a partir de material parental sedimentar (Almeida et al., 1997; Soil Survey Staff, 1999). Segundo Schaetzl (1998), partículas com diâmetro inferior a $30 \mu \mathrm{m}$ podem ser consideradas móveis no interior do perfil e passíveis de migração de horizontes superficiais ao horizonte subsuperficial, B. Para isso, uma seqüência de subprocessos deve ser desencadeada: desagregação de partículas umas das outras; transporte para horizontes subsuperficiais adjacentes; deposição nas paredes dos poros de horizontes subsuperficiais (Soil Survey Staff, 1999). Segundo Nooren et al. (1995) e Phillips (2007), a migração de argila em profundidade pode ser facilitada pela ação biológica seja pela bioturbação, seja pela formação de agregados frágeis à desagregação. Rousseau et al. (2004), ao estudarem os fatores físicos e químicos relacionados com a mobilidade de partículas coloidais no solo, verificaram que a condição inicial de umidade do solo, a intensidade do fluxo de água no interior do solo, o cátion saturante e a força iônica da solução do solo interferem no processo. Esses autores observaram também que a desagregação entre as partículas está ligada às propriedades hidrodinâmicas do solo, onde a força iônica da solução do solo constitui fator determinante da interação partícula-água.

Interrogações ainda subsistem, principalmente acerca das entidades mineralógicas envolvidas no processo e as conseqüências da migração para as propriedades gerais do solo. Nesse sentido, estima-se que a composição mineralógica da fração fina pode responder diferentemente ao processo de migração, considerando as propriedades intrínsecas de cada espécie mineral. A caracterização dos constituintes envolvidos no processo de migração, identificação mineralógica e distribuição do tamanho de partículas, tem importância básica para subsidiar futuros estudos em pedologia, contaminação de solo e água e fertilidade (Elimelech \& Ryan, 2002; Laegdsmand et al., 2007).

Deste modo, o presente estudo teve por objetivo avaliar a natureza mineralógica das partículas envolvidas no processo de formação de gradiente textural de um Argissolo subtropical do sul do Brasil localizado sob duas posições no relevo.

\section{MATERIAL E MÉTODOS}

\section{Localização do sítio e características do solo}

Dois perfis de solo foram estudados na área do Departamento de Solos da Universidade Federal de Santa Maria. O primeiro, perfil 1, localizado em meia encosta de uma colina (9\% de declividade) está sob campo nativo, vegetação nativa da região; o segundo, perfil 2 , de mesma classificação, está situado no topo da colina (0-1\% de declividade) e sob vegetação espontânea desde a década de 70 , quando foi abandonada a prática agrícola na área. $\mathrm{O}$ solo em ambos os perfis é classificado, segundo Embrapa (2006) como Argissolo Vermelho-Amarelo distrófico abrúptico textura franco-arenosa/argila, com transição abrupta entre os horizontes E e Bt e clara entre AB e Bt. Ambos os horizontes $\mathrm{Bt}$ apresentam cerosidade comum e moderada. Amostras de solo com estrutura preservada foram coletadas em 2002 nos horizontes $\mathrm{A}_{1}, \mathrm{~A}_{2}, \mathrm{AB}$ e $\mathrm{Bt}$ do perfil 1 e $\mathrm{A}_{1}, \mathrm{~A}_{2}, \mathrm{E}$ e Bt do perfil 2.

O material parental é sedimentar da Formação Santa Maria (Maciel Filho, 1988). Esta formação é composta pelos membros Alemoa e Passo das Tropas. Em geral, a litologia é representada por um arenito de médio a grosseiro, camadas de arenito síltico, siltito argiloso, compacto, maciço e de cor vermelha (Maciel Filho, 1988). A precipitação média anual da região é de $1.769 \mathrm{~mm}$ e a temperatura média anual de $19,2^{\circ} \mathrm{C}$, o que caracteriza um clima subtropical do tipo Cfa, segundo a classificação de Köeppen. Amostras do material de origem foram coletadas em corte de estrada representando cada perfil de solo.

\section{Análises químicas e físicas}

A capacidade de troca de cátions efetiva $\left(\mathrm{CTC}_{\mathrm{E}}\right)$ foi determinada pela soma dos cátions $\mathrm{Ca}, \mathrm{Mg}$ e $\mathrm{Al}$, extraídos por solução de $\mathrm{KCl} 1 \mathrm{~mol} \mathrm{~L}^{-1}$, e K, extraído por Mehlich-1; a CTC a pH $7\left(\mathrm{CTC}_{7}\right)$ foi calculada pela soma de $\mathrm{Ca}+\mathrm{Mg}+\mathrm{K}$ mais $\mathrm{H}+\mathrm{Al}$, que, por sua vez, foi estimado pelo $\mathrm{pH}$ SMP (Shoemaker et al., 1961; CQFSRS/SC, 2004). O carbono orgânico total (COT) foi determinado por combustão a seco com um analisador elementar, modelo C\&N Fison Carlo Erba. A determinação da textura (frações areia total, areia grossa, areia fina, silte e argila) e da densidade do solo (Ds), pelo método do anel volumétrico foi segundo Embrapa (1997). Determinou-se a densidade de partículas (Dp) pelo método do balão volumétrico. A porosidade total $(\mathrm{Pt})$ do solo foi calculada utilizandose densidade de partículas e do solo (Quadro 1). A área superficial específica (ASE) foi estimada gravimetricamente com uso de etilenoglicol mono etil éter (EGME), segundo Carter et al. (1965). A fim de identificar o caráter argilúvico, dois índices foram calculados, o primeiro, índice de diferenciação textural de horizontes (IDTh) que é a razão entre o teor de argila de um horizonte qualquer e aquele que apresenta o menor teor (Baize, 1988); o outro é a razão 
entre a fração menor que $0,2 \mu \mathrm{m}$ e a argila total de determinado horizonte (Soil Survey Staff, 1999; Hopkins \& Franzen, 2003).

Para determinar a presença de descontinuidade litológica no perfil, calcularam-se o valor de uniformidade (VU) adaptado de Schaetzl (1998) e a razão entre frações granulométricas areia fina:areia total. O VU é obtido pela razão entre a granulometria de um horizonte superficial (numerador) e a do horizonte subjacente (denominador) onde valores próximos a 1 denotam descontinuidade litológica entre os horizontes. A equação pode ser visualizada a seguir:

$$
\begin{gathered}
\mathrm{VU}=\left\{[(\mathrm{S}+\mathrm{AF}) /(\mathrm{A}-\mathrm{AF})]_{\text {horizonte superficial }} /\right. \\
\left.[(\mathrm{S}+\mathrm{AF}) /(\mathrm{A}-\mathrm{AF})]_{\text {horizonte subjacente }}\right\}-1,0
\end{gathered}
$$

em que VU é o valor de uniformidade; S é a fração silte, em percentagem; A é a fração areia, em percentagem; e $\mathrm{AF}$ é a fração areia fina, em percentagem.

Quadro 1. Atributos físicos e químicos de horizontes de um Argissolo Vermelho-Amarelo distrófico abrúptico, perfil 1, localizado em meia encosta sob campo nativo e perfil 2 , em topo de coxilha sob vegetação

\begin{tabular}{|c|c|c|c|c|c|c|c|c|}
\hline \multirow{2}{*}{$\begin{array}{l}\text { Local } \\
\text { Horizonte }\end{array}$} & \multicolumn{4}{|c|}{ Perfil 1 (meia encosta) } & \multicolumn{4}{|c|}{ Perfil 2 (topo de colina) } \\
\hline & A1 & A2 & AB & Bt & A1 & A2 & $\mathbf{E}$ & Bt \\
\hline Profundidade, m & $-0,42$ & $0,42-0,60$ & $0,62-0,80$ & $0,8+$ & $0-0,24$ & $0,24-0,62$ & $0,62-1,0$ & $1,0+$ \\
\hline Cor de Munsell & $\mathrm{YR} 3 / 2$ & 10YR3,5/3 & $7,5 \mathrm{YR} 4 / 4$ & $5 \mathrm{YR} 4,5 / 8$ & $10 Y R 3 / 3$ & 10YR3/2 & $10 \mathrm{YR} 4 / 3$ & $5 \mathrm{YR} 4 / 4$ \\
\hline \multicolumn{9}{|l|}{ Granulometria do solo ${ }^{(1)}$} \\
\hline Argila, $\mathrm{g} \mathrm{kg}^{-1}$ & 161 & 186 & 235 & 468 & 118 & 128 & 95 & 420 \\
\hline Silte, $\mathrm{g} \mathrm{kg}^{-1}$ & 248 & 221 & 211 & 184 & 206 & 230 & 374 & 172 \\
\hline Areia, $\mathrm{g} \mathrm{kg}^{-1}$ & 591 & 590 & 554 & 348 & 676 & 642 & 531 & 408 \\
\hline Areia grossa & 227 & 223 & 213 & 104 & 279 & 184 & 113 & 145 \\
\hline Areia fina & 364 & 367 & 341 & 244 & 397 & 458 & 418 & 263 \\
\hline Relação areia fina:total & 0,61 & 0,62 & 0,61 & 0,70 & 0,59 & 0,71 & 0,78 & 0,64 \\
\hline $\mathrm{COT}^{(2)}, \mathrm{g} \mathrm{kg}^{-1}$ & 74 & 66 & 69 & 56 & 98 & 70 & 30 & 53 \\
\hline Razão $0,2 \mu \mathrm{m}: 2 \mu \mathrm{m}^{(3)}$ & 0,23 & 0,29 & 0,34 & 0,50 & 0,14 & 0,14 & 0,09 & 0,41 \\
\hline $\mathrm{VU}^{(4)}$ & 0,02 & 0,02 & $-0,37$ & - & $-0,42$ & $-0,46$ & 1,33 & - \\
\hline $\operatorname{IDTh}^{(5)}$ & 1,00 & 1,20 & 1,50 & 2,90 & 1,20 & 1,40 & 1,00 & 4,40 \\
\hline \multicolumn{9}{|c|}{ Proporção de argilominerais da fração argila ${ }^{(6)}$} \\
\hline Caulinita-Esmectita & + & + & + & ++ & + & + & + & ++ \\
\hline Caulinita & * & $*$ & * & * & $*$ & * & * & * \\
\hline Esmectita-Ilita & $* *$ & $* * *$ & $* *$ & $* *$ & $* * *$ & $* * *$ & $* * *$ & $* *$ \\
\hline $\begin{array}{l}\text { Vermiculita hidróxi-Al } \\
\text { entrecamadas }\end{array}$ & $* *$ & * & $* *$ & * & * & * & * & * \\
\hline Ilita & * & * & * & * & * & * & * & * \\
\hline \multicolumn{9}{|l|}{ Propriedades físicas } \\
\hline $\mathrm{ASE}^{(7)}, \mathrm{m}^{2} \mathrm{~g}^{-1}$ & 131 & 136 & 137 & 138 & 97 & 105 & 87 & 126 \\
\hline Densidade solo $^{(8)}, \mathrm{kg} \mathrm{dm}^{-3}$ & 1,52 & 1,51 & 1,46 & 1,33 & 1,31 & 1,53 & 1,61 & 1,54 \\
\hline Densidade de partículas ${ }^{(9)}, \mathrm{kg} \mathrm{dm}^{-3}$ & 2,61 & 2,66 & 2,65 & 2,67 & 2,64 & 2,63 & 2,67 & 2,65 \\
\hline Porosidade total ${ }^{(10)}, \mathrm{m}^{3} \mathrm{~m}^{-3}$ & 0,420 & 0,434 & 0,449 & 0,503 & 0,504 & 0,420 & 0,395 & 0,417 \\
\hline \multicolumn{9}{|l|}{ Propriedades químicas } \\
\hline $\mathrm{pH} \mathrm{H} \mathrm{H}_{2} \mathrm{O}$ & 5,1 & 5,0 & 4,5 & 4,4 & 4,7 & 4,4 & 4,6 & 4,6 \\
\hline Saturação por bases, \% & 24 & 16 & 21 & 18 & 53 & 27 & 22 & 44 \\
\hline $\mathrm{CTC}_{\mathrm{E}}{ }^{(11)}, \mathrm{cmol}_{\mathrm{c}} \mathrm{kg}^{-1}$ & 2,5 & 2,3 & 4,2 & 7,3 & 3,1 & 2,4 & 1,3 & 6,8 \\
\hline $\mathrm{CTC}_{7}{ }^{(12)}, \mathrm{cmol}_{\mathrm{c} \mathrm{kg}}{ }^{-1}$ & 2,7 & 2,4 & 6,4 & 10,4 & 4,6 & 3,5 & 2,6 & 9,1 \\
\hline
\end{tabular}
pioneira, Santa Maria-RS, 2008

${ }^{(1)}$ Argila menor que $2 \mu \mathrm{m}$, Silte de 2 a $50 \mu \mathrm{m}$ e Areia de 50 a $2.000 \mu \mathrm{m} .{ }^{(2)}$ COT: Carbono orgânico total. ${ }^{(3)}$ Razão entre a fração menor que $0,2 \mu \mathrm{m}$ e a argila total (Soil Survey Staff, 1999). ${ }^{(4)}$ VU: refere-se a um valor de uniformidade. ${ }^{(5)}$ IDTh: refere-se à razão entre o teor de argila de um horizonte qualquer e o teor do horizonte com menor teor. ${ }^{(6)}$ Proporção segundo a escala: * 1 -10 \%;** 11-25 \%;*** 26-40 \%;+ 41-65 \% e ++ 66-80\% (Bortoluzzi, 2003). ${ }^{(7)}$ ASE: Área Superficial Específica. ${ }^{(8)}$ Ds: estimado por anel volumétrico, segundo Embrapa (1997). ${ }^{(9)}$ Dp: estimado pelo método do balão volumétrico, segundo Embrapa (1997). (10) Pt: determinado pela equação: $\mathrm{Pt}=(\mathrm{Dp}-\mathrm{Ds}) / \mathrm{Dp} .{ }^{(11)} \mathrm{CTC}_{\mathrm{E}}$ : capacidade de troca de cátions efetiva. ${ }^{(12)} \mathrm{CTC}_{7}$ : capacidade de troca de cátions a $\mathrm{pH} 7$. 
As características gerais do solo, assim como os índices calculados, estão apresentados no quadro 1.

As amostras de solo e rocha da fração $<2 \mathrm{~mm}$ foram submetidas ao ataque ácido com $\mathrm{HF}$ e os elementos totais $\mathrm{Ca}, \mathrm{Mg}, \mathrm{K}, \mathrm{Na}, \mathrm{Fe}$ total medidos por absorção atômica. Si, Al e Ti foram solubilizados por fusão alcalina e medidos por fluorescência X (Afnor, 1996). O teor de Fe livre foi obtido segundo Mehra \& Jackson (1960). A concentração dos elementos totais foi corrigida para a massa da amostra a $105^{\circ} \mathrm{C}$ e seus resultados expressos em percentagem mássica de seus respectivos óxidos $\left(\mathrm{CaO}, \mathrm{MgO}, \mathrm{K}_{2} \mathrm{O}, \mathrm{Na}_{2} \mathrm{O}, \mathrm{Fe}_{2} \mathrm{O}_{3} \mathrm{e}\right.$ $\mathrm{Al}_{2} \mathrm{O}_{3}$ ). Em seguida cada valor foi equalizado à concentração de titânio do seu horizonte e àquela encontrada na rocha, equação 2 . Já a proporção dos elementos em relação à rocha foi obtida através da aplicação da equação 3. Os resultados foram então expressos em porcento de isotitânio da concentração existente na rocha.

$$
M_{\mathrm{Hz}}=\left(M \mathrm{x} \mathrm{Ti}_{\text {Rocha }}\right) / \mathrm{Ti}_{\mathrm{Hz}}
$$

em que $M_{H z}$ é a proporção isotitânio de um elemento em dado horizonte de solo; $M$ é a proporção de determinado elemento no horizonte considerado; $\mathrm{Ti}_{\text {Rocha }}$ é a proporção de titânio na rocha em relação aos outros óxidos, e $\mathrm{Ti}_{\mathrm{Hz}}$ proporção de titânio no horizonte considerado.

$$
M p=\left(M_{H z} / M_{\text {Rocha }}\right) \times 100
$$

em que $M p$ é a percentagem de um elemento em dado horizonte em relação à rocha; $M_{\text {Rocha }}$ é a concentração isotitânio de determinado elemento na rocha.

\section{Análises mineralógicas}

Em amostras de $25 \mathrm{~g}$ da fração menor que $2 \mathrm{~mm}$, destruiu-se a matéria orgânica por queima com $\mathrm{H}_{2} \mathrm{O}_{2}$, submetendo-as em seguida à dispersão sob agitação constante, adicionando-se $5 \mathrm{~mL}$ da solução $\mathrm{NaOH}$ $2,5 \mathrm{~mol} \mathrm{~L}^{-1}$ e água deionizada para completar $200 \mathrm{~mL}$ de suspensão. A separação granulométrica foi realizada por peneiramento para extração da fração areia, sedimentação para a separação da fração silte e centrifugações para a separação da fração menor que 2 e $0,2 \mu \mathrm{m}$ (Robert \& Tessier, 1974). O material dessas frações foi saturado com $\mathrm{CaCl}_{2}$ e $\mathrm{KCl}$, sendo, posteriormente, lavado para eliminar o excesso dos íons.

Suspensões da fração entre 2 e $5 \mu \mathrm{m}$ e menor que $0,2 \mu \mathrm{m}$ foram preparadas em depósito orientado, pipetagem do material sobre lâminas de vidro e submetidas aos seguintes tratamentos: (a) secas em temperatura ambiente, normal $(\mathrm{N})$; (b) solvatadas com etilenoglicol (EG); (c) aquecidas a 300 e $550^{\circ} \mathrm{C}$. Nessas lâminas, difratogramas de raios X (DRX) foram obtidos com amplitude angular de $2^{\circ}$ a $35^{\circ}$ de $2 \theta$ para as amostras $(\mathrm{N})$, e de $2^{\circ}$ a $16^{\circ}$ de $2 \theta$ para os outros tratamentos. A identificação dos minerais seguiu a ocorrência de picos correspondentes ao plano 001, segundo Brindley \& Brown (1980). O difratômetro utilizado foi de modelo Siemens D5000, equipado com um monocromador, operando com radiação $\mathrm{CoK} \alpha$ a $40 \mathrm{kV}$ de voltagem e uma corrente elétrica de $30 \mathrm{~mA}$. Uma análise semiquantitativa dos argilominerais foi realizada sobre os DRX com auxílio do programa de computador DecompRX (Lanson, 1997). Para isso, os picos correspondentes ao plano 001 foram separados em fases elementares, representados por curvas do tipo gaussiana ou lorenciana para cada espécie mineral presente. Obteve-se DRX modelado, bem como a respectiva área relativa de cada fase mineral da fração estudada, conforme Lanson (1997).

Adicionalmente, uma alíquota da fração menor que $2 \mu \mathrm{m}$, em presença de hexamatafosfato de sódio, foi submetida à difração laser. Um difratômetro laser modelo Coulter LS230 do INRA de Versailles com tecnologia PIDS foi utilizado. Os limites de detecção do equipamento são de 0,04 a $2.000 \mu \mathrm{m}$ de diâmetro de partículas. Nesse estudo, o equipamento permitiu a separação da fração $2 \mu \mathrm{m}$ em 116 classes de tamanho de partículas. Detalhes do princípio de funcionamento podem ser encontrados em Dur et al. (2004) e Bortoluzzi \& Poleto (2006). Os resultados foram apresentados na forma de diagramas de difração laser (DDL), em que a distribuição do tamanho de partículas representa a freqüência em percentual do volume que cada classe de diâmetro das partículas aparece na amostra.

\section{RESULTADOS E DISCUSSÃO}

No quadro 1 , a $\mathrm{CTC}_{7}$ variou em ordem crescente de 2,4, 2,7, 6,4 e 10,4 $\mathrm{cmol}_{\mathrm{c}} \mathrm{kg}^{-1}$, respectivamente, para os horizontes $\mathrm{A}_{2} \mathrm{~A}_{1}, \mathrm{AB}$ e $\mathrm{Bt}$ do perfil 1. Para o perfil 2, a $\mathrm{CTC}_{7}$ variou de 4,6, 3,5, 2,6 e 9,1 $\mathrm{cmol}_{\mathrm{c}} \mathrm{kg}^{-1}$, respectivamente, para os horizontes $\mathrm{A}_{1}, \mathrm{~A}_{2}, \mathrm{AB}$ e Bt. $\mathrm{A} \mathrm{CTC}_{\mathrm{E}}$ variou inversamente com o $\mathrm{pH}$, apresentando, para ambos os perfis, valores levemente mais baixos que a $\mathrm{CTC}_{7}$. Isto concorda com a ASE da fração argila que variou de 87 a $138 \mathrm{~m}^{2} \mathrm{~g}^{-1}$, valores compatíveis com a presença de argilominerais do tipo 2:1. Os teores de COT, para o perfil 1 , variaram para os horizontes $\mathrm{A}_{1}$, $\mathrm{A}_{2}, \mathrm{AB}$ e Bt, respectivamente $\left(74,66,69\right.$ e 56) $\mathrm{g} \mathrm{kg}^{-1}$, enquanto, para o perfil 2 , os valores foram de $(98,70$, 30 e 53) $\mathrm{g} \mathrm{kg}^{-1}$, para os respectivos horizontes. O teor de COT do Bt representou, no mínimo, cerca de $50 \%$ dos teores dos demais horizontes.

Para o teor de argila, observa-se através do IDTh comportamento distinto entre os horizontes para ambos os perfis de solo. A relação entre o teor de argila dos horizontes $\mathrm{A}_{2}, \mathrm{AB}$ e $\mathrm{Bt}$ do perfil 1 para o horizonte com menor teor de argila $\mathrm{A}_{1}$ é, respectivamente, 1,2, 1,5 e 2,9 vezes. Para o perfil 2, essa relação nos horizontes $\mathrm{A}_{1}, \mathrm{~A}_{2} \mathrm{e}$ Bt para o horizonte E é ainda maior, respectivamente, 1,2, 1,4 e 4,4 vezes. Isto indica a presença de forte gradiente textural, 
principalmente para o perfil 2, corroborando a presença de um horizonte Bt. A interpretação acerca da razão entre argila fina e argila total auxilia a identificação do processo de iluviação de partículas finas. Assim, quanto mais partículas finas na fração argila maior será a probabilidade de que estas tenham sido iluviadas. Dessa forma, observa-se, no perfil 1, para os horizontes $\mathrm{A}_{1}, \mathrm{~A}_{2}, \mathrm{AB}$ e $\mathrm{Bt}$, uma razão de 0,23 , $0,29,0,34,0,50$ e, no perfil 2 , para os horizontes $\mathrm{A}_{1}$, $\mathrm{A}_{2}$, E e Bt, uma razão de 0,14, 0,14, 0,09 e 0,41, respectivamente. Nos horizontes superficiais, a fração menor que 0,2 $\mu \mathrm{m}$ compõe a fração argila em, no máximo, $34 \%$, enquanto para os horizontes Bt chega a 50 \%, indicando provável migração (Quadro 1).

$\mathrm{O}$ valor de uniformidade VU, se superior a 0,6 , oferece indícios de descontinuidade litológica, caso contrário, indica que o perfil de solo foi desenvolvido a partir do mesmo material de origem. Esses dados auxiliam na interpretação da origem do gradiente textural do perfil de solo, visto que a descontinuidade litológica também pode produzi-lo (Schaetzl, 1998). Observam-se, no perfil 1, índices VU menores que 0,6 , indicando continuidade litológica, logo um perfil autóctone (Quadro 1). Já, para o perfil 2, um valor de 1,33 foi encontrado entre o horizonte $\mathrm{E}$ e Bt, evidenciando descontinuidade litológica entre esses horizontes, logo um perfil alóctone.

Entretanto, outras evidências são necessárias para confirmar a interpretação de descontinuidade litológica (Schaetzl, 1998). A razão entre a areia fina e total também pode ser utilizada. Nesse sentido, para o perfil 1, a razão apresenta valores ao redor de 0,60 para os horizontes superficiais e de 0,70 para o Bt; enquanto, para o perfil 2, os valores ficam entre 0,59 e 0,78 para os horizontes superficiais e de 0,64 para o Bt (Quadro 1). Observa-se com esta relação que a areia fina é predominante na fração areia total e os valores denotam certa uniformidade entre os horizontes, com amplitudes não superiores a 0,10 , para o perfil 1, e 0,19, para o perfil 2. Valores similares aos observados no presente trabalho foram obtidos por
Almeida et al. (1997), e Mafra et al. (2001) indicando ausência de descontinuidade litológica nos perfis de solo estudados.

Quanto à mineralogia da fração argila, encontrase, no quadro 1, a proporção das espécies nos horizontes estudados. Nessa fração, foram encontradas as seguintes espécies argilominerais: ilita com distância basal (d) de 1,00 nm, caulinita ( $d=0,717 \mathrm{~nm})$, ilitaesmectita I-E $(\mathrm{d}=1,439 \mathrm{~nm})$ e interestratificado caulinita-esmectita $\mathrm{C}$-E representado por um pico superior a $d=0,717 \mathrm{~nm}$ (Bortoluzzi, 2003). A presença de C-E é evidenciada quando o pico a $\mathrm{d} \approx 0,72 \mathrm{~nm}$ é sensível à solvatação com EG e ao aquecimento (Moore \& Reynolds, 1997; Bortoluzzi et al., 2007). A vermiculita com hidróxi-Al entrecamada (VHE) foi identificada através da presença de um pico a $\mathrm{d}=1,400 \mathrm{~nm}$ no DRX a $25^{\circ} \mathrm{C}$, quando não houve expansão com EG e quando aquecida a 300 e $550{ }^{\circ} \mathrm{C}$ provocou o fechamento incompleto das entrecamadas próximo a $\mathrm{d}=1,00 \mathrm{~nm}$, mesmo saturadas com K. A fase I-E foi identificada quando do aparecimento de um pico largo entre 1,40 e $1,02 \mathrm{~nm}$, sensível ao tratamento com EG e o aquecimento a $550{ }^{\circ} \mathrm{C}$ deslocando-se a $1,0 \mathrm{~nm}$.

$\mathrm{Na}$ figura 1, observa-se, em geral, que a quantidade de elementos totais foi inferior a $50 \%$ nos horizontes superficiais do perfil 1 (posição de declive) daquela encontrada no material parental, enquanto, para o perfil 2, foi de cerca de $25 \%$. Para o perfil 1 , e no caso do $\mathrm{CaO}$, o horizonte $\mathrm{A}_{1}$ apresentou maior proporção, cerca de $45 \%$, diminuindo para os outros horizontes, variando entre 20 e $25 \%$. Valores muito similares também foram encontrados para o $\mathrm{MgO}$. Os valores para $\mathrm{K}_{2} \mathrm{O}$ e $\mathrm{Na}_{2} \mathrm{O}$ em relação aos da rocha foram sempre abaixo de $8 \%$, em virtude do caráter mais móvel dos elementos. Os valores para $\mathrm{MgO}$, $\mathrm{Al}_{2} \mathrm{O}_{3}$, Fe livre e total em relação aos da rocha tiveram a tendência de aumento com a profundidade dos horizontes, para ambos os perfis. E importante notar que a rocha é sedimentar composta por siltitos e arenitos, logo, um material friável e permeável, o que pode explicar a presença de Fe livre. Para o horizonte
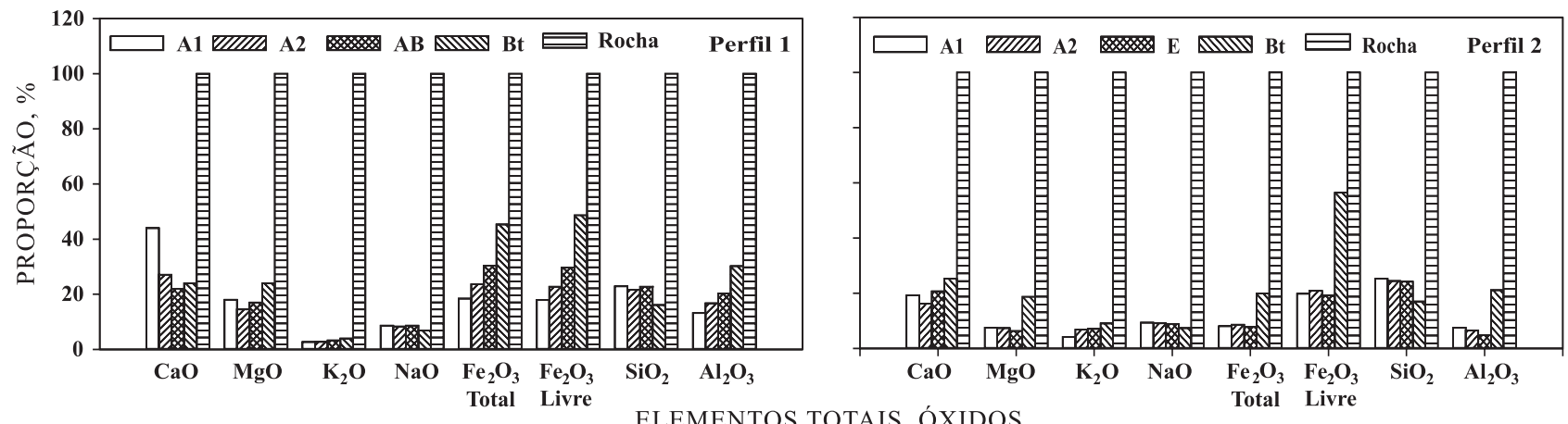

Figura 1. Proporção, em relação ao material de origem, de elementos totais presentes nos horizontes de um Argissolo Vermelho-Amarelo distrófico abrúptico, perfil 1, localizado em meia encosta sob campo nativo e perfil 2, em topo de coxilha sob vegetação pioneira. 
Bt do perfil 1, os mesmos elementos foram, respectivamente, $25,30,50$ e $45 \%$ do encontrado no material de origem, enquanto, para o perfil 2 , foram, respectivamente, cerca de $18,20,55$ e $20 \%$.

No perfil 1, a proporção dos elementos em relação ao material parental mostrou-se com um gradiente mais uniforme que no perfil 2 . Isto concorda com a presença de um horizonte $\mathrm{AB}$, logo, um gradiente textural mais suave nesse perfil com teores de argila intermediários entre os horizontes superficiais e o Bt. Para o perfil 2, o horizonte E, mais arenoso que os demais, determina valores mais baixos de elementos totais. A maior proporção dos elementos totais nos horizontes subsuperficiais pode ser indicativo da destruição de argilominerais por ferrólise, como o observado por Mafra et al. (2001). A formação do horizonte $\mathrm{E}$, no perfil 2, poderia ser conseqüência desse processo, porém não de forma isolada, atuando conjuntamente com o processo de eluviação de argila. A razão entre as frações menor que $0,2 \mu \mathrm{m}$ e argila corrobora essa idéia. O indicativo de gradiente textural das partículas finas pode confirmar o processo de migração de argila.

Nesse sentido, na figura 2, estão apresentados os diagramas de difração laser (DDL) oriundos da fração argila dos horizontes de solo dos perfis 1 e 2 . Observase, em todos os horizontes dos perfis, a existência de quatro classes modais de tamanho de partículas. Dois picos de grande intensidade foram encontrados no DDL, correspondendo aos diâmetros modais de partículas de $0,09 \mu \mathrm{m}$ e $0,8 \mu \mathrm{m}$, e dois de menor intensidade, correspondendo aos diâmetros de $0,25 \mathrm{e}$ $2 \mu \mathrm{m}$. No perfil 1, a proporção das partículas de tamanho $<0,2 \mu \mathrm{m}$ aumenta gradativamente com a profundidade. Essa classe de partículas representa cerca de $20 \%$ do total na fração argila do horizonte $\mathrm{A}_{1}$, cerca de $30 \%$ nos horizontes $\mathrm{A}_{2}$ e $\mathrm{AB}$ e mais da metade no horizonte $\mathrm{Bt}$, em detrimento das partículas de diâmetro entre 0,2 e $2 \mu \mathrm{m}$. No perfil 2 , observa-se que as partículas menores que $0,2 \mu \mathrm{m}$ representam menos que $14 \%$ nos horizontes superficiais e cerca de $41 \%$ no Bt do total da fração argila (Quadro 1 e Figura 2).

Esses resultados indicam que os perfis têm caráter argilúvico, de acordo com a presença de horizonte $\mathrm{Bt}$ e interpretações de Hopkins \& Franzen (2003). Observa-se, no horizonte Bt, um enriquecimento em partículas de tamanho argila, e, por sua vez, essa fração é rica em partículas finas (menor que $0,2 \mu \mathrm{m}$ ). Essas partículas, provavelmente, são responsáveis pela cerosidade verificada no horizonte Bt de ambos os perfis. Pode-se inferir com segurança que o processo de translocação vertical de partículas é marcante na formação de gradiente textural dos perfis. Isto também é em acordo com interpretações realizadas por Almeida et al. (1997) em uma catena de solos do Rio Grande do Sul. É importante notar que as classes de tamanho de partículas da fração argila são muito similares em seus diâmetros modais, indicando um material parental também muito similar para os perfis. Tais

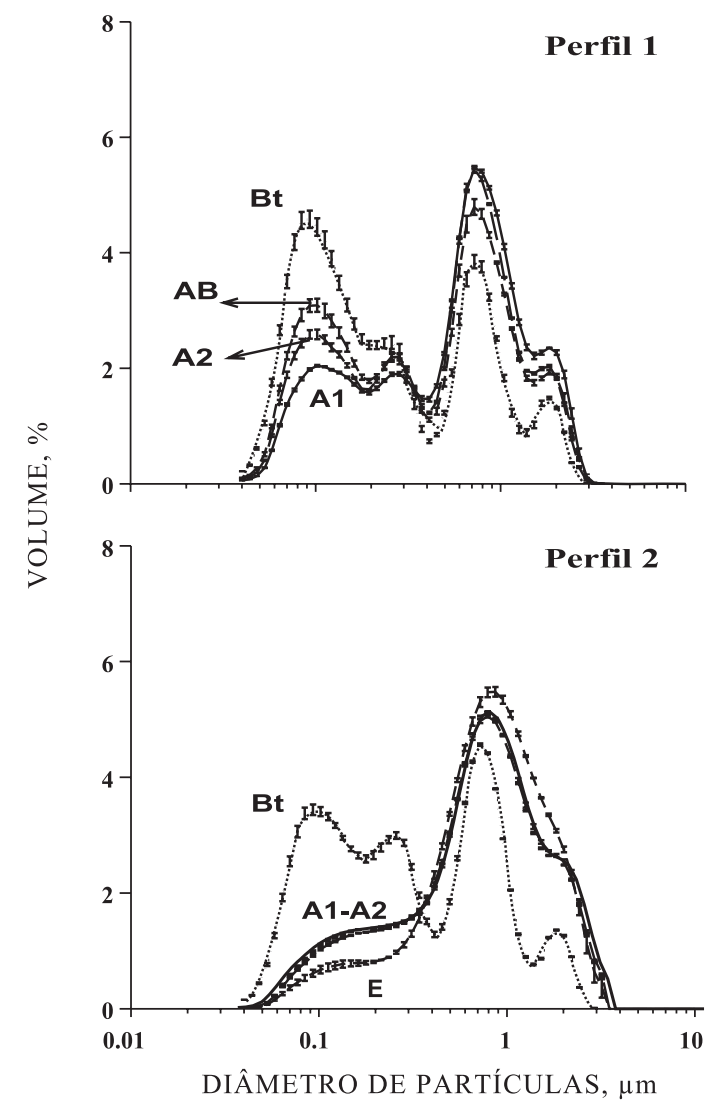

Figura 2. Diagramas de difração laser representando a distribuição do tamanho de partículas em percentual de seus volumes em cada classe. Fração argila dos horizontes de um Argissolo Vermelho-Amarelo distrófico abrúptico, perfil 1, localizado em meia encosta sob campo nativo e perfil 2 , em topo de coxilha sob vegetação pioneira.

resultados não confirmam a descontinuidade litológica indicada para o perfil 2 através do índice VU.

Na figura 3, os difratogramas de raios X (DRX) da fração menor que $0,2 \mu \mathrm{m}$ são apresentados. Neles, observam-se picos a $d=1,439 \mathrm{~nm}$ e $d=0,730 \mathrm{~nm}$ menos intensos para os horizontes subsuperficiais Bt que para os horizontes superficiais $A_{1}$. Isso denota uma variação na proporção relativa dos argilominerais. No entanto, a cristalinidade dos argilominerais pode ser considerada similar, visto que é observada na largura do pico a meia altura. Nesse sentido, encontram-se, na figura 4, os DRX modelados da fração menor que $0,2 \mu \mathrm{m}$. Nesta fração, foi possível identificar e quantificar a presença de somente dois grupos de argilominerais; o primeiro, correspondente à região próxima a $\mathrm{d}=1,440 \mathrm{~nm}$, e o outro, próximo a $\mathrm{d}=0,730 \mathrm{~nm}$, podendo-se atribuir, respectivamente, à presença de I-E e VHE e de interestratificado C-E. Caulinita $(\mathrm{d}=0,717 \mathrm{~nm})$ e ilita $(\mathrm{d}=1,00 \mathrm{~nm})$ não foram observadas nessa fração, assim como o quartzo. Kampf et al. (1995) consideram comum a presença de VHE em solos do Rio Grande do Sul. 
Na figura 3, observa-se ainda que a fração entre 2-5 $\mu \mathrm{m}$ apresenta DRX com picos menos intensos, principalmente os do horizonte superficial do perfil 2 . Já para a amostra do Bt, o solo apresentou um pequeno pico referente à mica, $d=1,00 \mathrm{~nm}$, inexistente nos outros diagramas. Nessa fração, o quartzo apresenta-se nos DRX com picos intensos.

Observa-se, na figura 4, uma proporção de C-E superior no horizonte $B t$ que no horizonte $A_{1}$ em detrimento das duas fases a $\mathrm{d}=1,40 \mathrm{~nm}$. No horizonte $\mathrm{A}_{1}$ do perfil 1, a intensidade e a largura a meia altura dos picos são similares, o que resulta em uma área com proporção similar, $47 \%$ para o pico $d=1,438 \mathrm{~nm}$ referente a I-E e $53 \%$ para o pico $d=0,730 \mathrm{~nm}$ atribuído a C-E. Para o horizonte Bt do mesmo perfil, existe a tendência de maior proporção da fase $\mathrm{d}=$ 0,730 nm, com 58,8 \%, em detrimento de 41,1\% do pico $d=1,414 \mathrm{~nm}$. Para o perfil 2, o pico a $d=1,400 \mathrm{~nm}$ para os horizontes $\mathrm{A}_{1}$ e $\mathrm{Bt}$ apresentou intensidade menor, resultando em uma proporção de 31,9 e 12,3\% que o pico a $d=0,730 \mathrm{~nm}$ da C-E com 68,1 e 87,7 \%, respectivamente.

Com esses resultados é possível afirmar que há um enriquecimento de argilominerais do tipo C-E no horizonte $\mathrm{Bt}$ em relação ao horizonte $\mathrm{A}_{1}$, resultante da migração preferencial destas partículas no interior do perfil. Ademais, o diâmetro modal das partículas envolvidas preferencialmente na migração é de

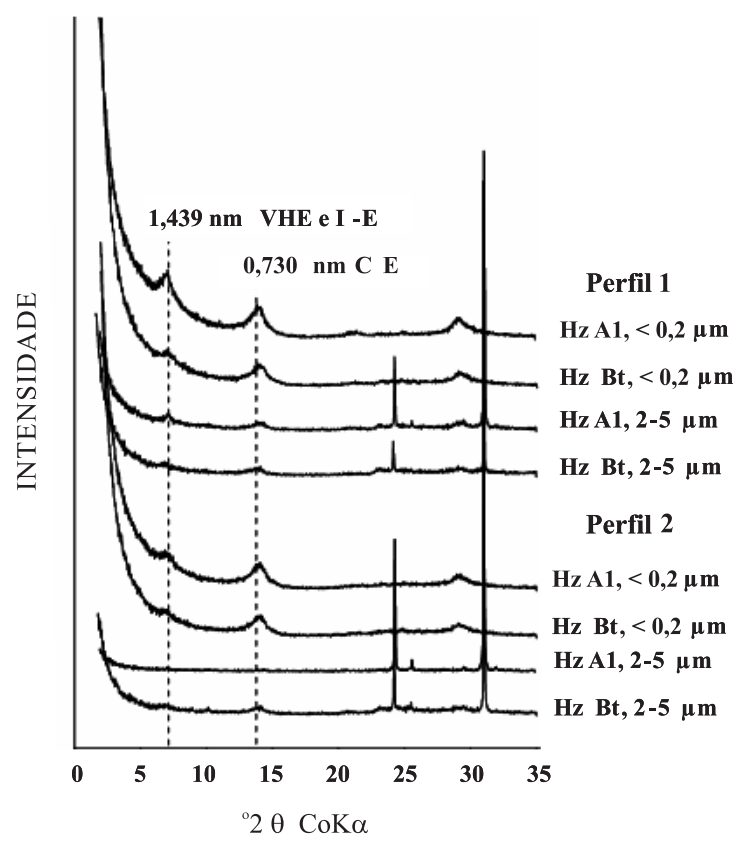

Figura 3. Difratogramas de raios $\mathrm{X}\left(25^{\circ} \mathrm{C}\right)$ da fração menor que $0,2 \mu \mathrm{m}$ e entre $2-5 \mu \mathrm{m}$, saturadas com $\mathrm{Ca}^{2+}$ dos horizontes $\mathrm{A}_{1}$ e Bt de um Argissolo Vermelho-Amarelo distrófico abrúptico, perfil 1, localizado em meia encosta sob campo nativo e perfil 2 , em topo de coxilha sob vegetação pioneira.
$0,09 \mu \mathrm{m}$, como representado na figura 2. Estudos de Dur et al. (2005) em solos tropicais da Tailândia associaram o tamanho modal de partículas a uma espécie de argilomineral envolvida no processo de migração. Os mesmos autores corroboram, ainda, a idéia de que associações entre as partículas que migram e óxidos contribuem para o incremento de $\mathrm{Fe}$ e Al na porção mais profunda do perfil de solo.

O horizonte Bt é, em relação aos superficiais, menos intemperizado, pois evolui próximo à camada $\mathrm{C}$ ou diretamente sobre o material de origem. O perfil 1, por não apresentar indícios de descontinuidade litológica, apresenta horizonte Bt enriquecido de partículas finas oriundas de horizontes superficiais mais intemperizados (Quadro 1 e Figura 2). Para o perfil 2, existe o indício de descontinuidade litológica entre o horizonte $\mathrm{E}$ e Bt, porém é um indício contraditório em relação a outros testados. Na hipótese de descontinuidade litológica, a conseqüência é que o gradiente textural presente nesse perfil seja produto, preponderantemente, da deposição pretérita de materiais aluviais, mais arenosos, sobre o material correspondente ao atual horizonte Bt (Schaetzl, 1998). Assim, se materiais parentais diferentes compõem o perfil 2, a mineralogia e a granulometria entre os horizontes deveriam ser também diferentes, fato este não verificado (Figuras 2, 3 e 4). Nesse sentido, a hipótese de migração de partículas de horizontes superficiais e formação de horizonte Bt como principal fator de gradiente textural aplica-se a ambos os Perfis.

Para o perfil 2, apenas o índice VU indicou descontinuidade litológica, outros indícios como a relação granulométrica entre argila fina e total e areia fina e total foram discordantes (Quadro 1). Alguns autores alertam para o fato de serem necessários vários parâmetros para discriminar com segurança a presença de descontinuidade litológica e a condição alóctone do perfil de solo (Schaetzl, 1998). Estudos, como o apresentado aqui, baseiam-se na natureza mineralógica (van Ranst \& De Coninck, 2002) e nas relações granulométricas (Oliveira et al., 2004) e oferecem maior segurança. Dessa forma, ambos os perfis mostraram-se similares quanto à natureza da fração fina da argila (Figura 3 e 4) e o tamanho modal das partículas, indicando ser material parental similar que originou ambos os perfis (Figura 2).

Quanto ao processo de ferrólise como promotor do gradiente textural, espera-se que seja atuante por ocasião de pH baixo, ao redor de 4 (Mafra et al., 2001). Para Almeida et al. (1997), o processo de ferrólise atuante na parte superior de horizontes B texturais, juntamente com a eluviação de argila, foi responsável pela formação de gradiente textural. Indícios na mineralogia, como a maior polimerização de $\mathrm{Al}$ entrecamadas de argilominerais 2:1 em direção ao topo do perfil, foram constatados por esses autores. Entretanto, as características ideais que indicam a eminente ação de ferrólise baseiam-se na presença em frações finas de clorita trioctaedral com $d=1,400 \mathrm{~nm}$ 
após aquecimento a $550{ }^{\circ} \mathrm{C}$. Para isso essa espécie deve ser originária de frações maiores como a do silte. A presença de quartzo na fração argila fina e o decréscimo de elementos totais em horizontes superficiais podem também indicar a atuação da ferrólise (van Ranst \& Coninck, 2002). No presente estudo, apesar de o pH ser naturalmente baixo, não foi constatada a presença de quartzo e clorita na fração menor que $0,2 \mu \mathrm{m}$. Nessa fração, observou-se a presença de quantidades superiores de VHE em superfície que no Bt (Figura 4), mas esta quantidade mostrou-se similar na fração argila do perfil (Quadro 1).

Quanto à formação de gradiente textural, não se pode excluir do raciocínio o processo de bioturbação estudado por Phillips (2007). Através de canais de origem biológica assembléias de argilominerais finos e de partículas organominerais oriundas da atividade de organismos como os anelídeos tornam-se passíveis à migração (Nooren et al., 1995). É notório que a atividade biológica do solo em climas subtropicais seja importante, visto que o regime de chuvas é bem distribuído ao longo do ano e a temperatura média da região de estudo é branda, ao redor de $19^{\circ} \mathrm{C}$. Estimase que a bioturbação auxilie o processo de migração de partículas finas e formação de gradientes texturais em Argissolos subtropicais. A migração de argila passa a ser, então, um processo dependente também do: clima, relevo, mineralogia e propriedades físicoquímicas do solo (Phillips, 2001).

No presente trabalho, somente o perfil 1 apresenta indícios de forte formação de horizonte Bt pelo processo de iluviação de argila. As partículas envolvidas na migração apresentam um diâmetro modal entre 0,09$0,2 \mu \mathrm{m}$ enquanto as partículas maiores com diâmetro modal próximo a $0,8 \mu \mathrm{m}$ mostraram-se menos sensíveis ao processo de migração (Figura 2). O perfil 2 revela indícios conflitantes quanto à presença de descontinuidade litológica. Apesar disso, no perfil 2, há indícios de uniformidade litológica indicados pela natureza mineralógica o que permite inferir sobre a translocação de partículas finas no seio do perfil pelo processo de iluviação-eluviação. No entanto, é provável que mais de um processo seja responsável pela formação
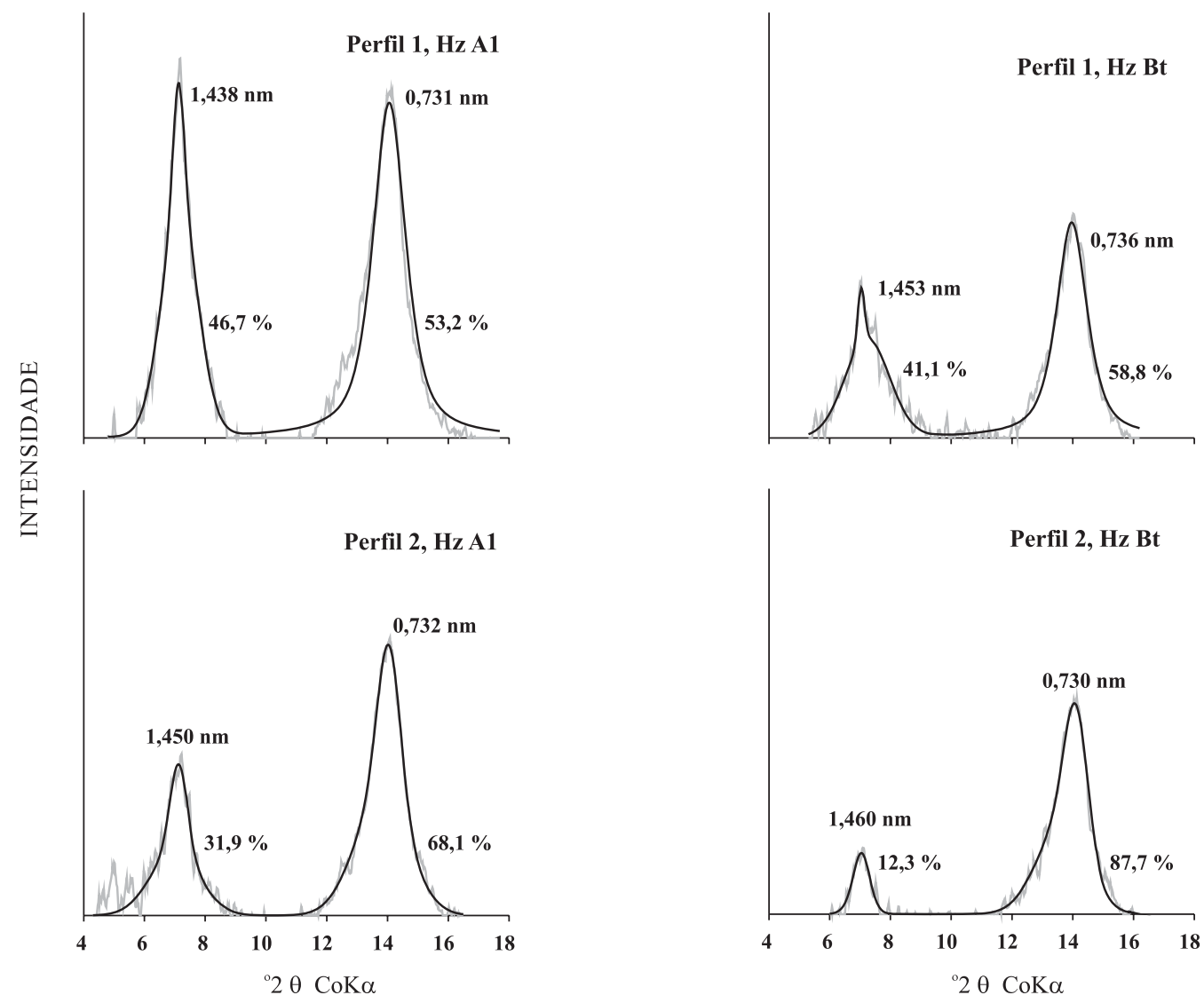

Figura 4. Difratogramas de raios $\mathrm{X}\left(25^{\circ} \mathrm{C}\right)$ entre os ângulos 4 e $18^{\circ}$ de $2 \theta$ após modelagem matemática com DecompXR, obtidos a partir de amostras da fração menor que $0,2 \mu \mathrm{m}$, saturadas ao $\mathrm{Ca}^{2+}$ do horizonte $\mathrm{A} 1$ e Bt de um Argissolo Vermelho-Amarelo distrófico, perfil 1, localizado em meia encosta sob campo nativo e perfil $2 \mathrm{em}$ topo de coxilha sob vegetação pioneira. A linha cheia clara ( $\longrightarrow$ refere-se à curva experimental do DRX, a linha cheia escura (_) refere-se à curva ajustada pelo DecompXR. 
de gradiente textural, como alertam Phillips (2001) e Phillips (2004).

$\mathrm{O}$ argilomineral interestratificado caulinitaesmectita de diâmetro modal 0,09 $\mu$ m está envolvido na formação do gradiente textural em ambos os perfis (Figuras 2 e 4). Dada a uniformidade apresentada em ambos os perfis do Argissolo quanto à mineralogia (Figuras 3 e 4) e à granulometria modal das partículas (Figura 2), o gradiente textural é provavelmente produto da ação predominante do processo de iluviaçãoeluviação de partículas. Para o perfil 2 , além da migração de argila, o processo de ferrólise mostra-se também bastante atuante, sendo sustentado pelos resultados dos teores totais de óxidos (Figura 1).

$\mathrm{O}$ fato de haver migração em subsuperfície de argilas de alta carga permanente e área superficial específica pode constituir um mecanismo de conservação de certos argilominerais em Argissolos (Pedro, 1979) e de redistribuição de contaminantes no perfil (Laegdsmand et al., 2007). Isso por que, em subsuperfície, os argilominerais 2:1 encontram condições termodinâmicas mais favoráveis à sua conservação, ao contrário das condições em superfície. Ademais, os atributos químicos e físicos estão em concordância com as características desses argilominerais, como, por exemplo, a contribuição majoritária das cargas elétricas permanentes à CTC dos horizontes subsuperficiais, como anteriormente verificado por Bortoluzzi et al. (2006).

\section{CONCLUSÕES}

1. O Argissolo Vermelho-Amarelo distrófico abrúptico apresentou forte gradiente textural em ambos os perfis estudados. O gradiente textural do perfil 1 foi originário do processo de iluviação de argila fina para o Bt. O perfil 2 apresentou, além da migração de argila como processo de formação de gradiente textural, provável descontinuidade litológica.

2. O interestratificado caulinita-esmectita de diâmetro modal de 0,09 e 0,2 $\mu \mathrm{m}$ foi o argilomineral envolvido no processo de migração de partículas e acúmulo de material no Bt do solo estudado.

\section{AGRADECIMENTOS}

Os autores agradecem ao CNPq pela concessão de Bolsa de Doutorado Pleno no exterior ao primeiro autor.

\section{LITERATURA CITADA}

AFNOR. Qualité des sols: Recueil des normes françaises. 3.ed. Paris, La Défense, 1996. 534p.
ALMEIDA, J.A.; KLAMT, E. \& KÄMPF, N. Gênese do contraste textural e da degradação do horizonte B de um Podzólico Vermelho-Amarelo da planície costeira do Rio Grande do Sul. R. Bras. Ci. Solo, 21:221-233, 1997.

BAIZE, D. Guide des analyses courantes en pédologie. Paris, INRA, 1988. 172p.

BORTOLUZZI, E.C. Nature des constituants, propriétés chimiques et physiques des sols. Modélisation des charges superficielles dans des sols sableux au sud du Brésil. Paris, INAPG, INRA, 2003. 145p. (Tese de Doutorado)

BORTOLUZZI, E.C. \& POLETO, C. Metodologias para estudos de sedimentos: ênfase na proporção e na natureza mineralógica das partículas. In: MERTEN, G.H. \& POLETO, C., orgs. Qualidade de sedimentos. Porto Alegre, ABRH, 2006. p.80-140

BORTOLUZZI, E.C.; PERNES, M. \& TESSIER, D. Interestratificado caulinita-esmectita em um Argissolo desenvolvido a partir de rocha sedimentar do sul do Brasil. R. Bras. Ci. Solo, 31:1291:1300, 2007.

BORTOLUZZI, E.C.; TESSIER, D.; RHEINHEIMER, D.S. \& JULIEN, J.L. The cation exchange capacity of a sandy soil $\mathrm{n}$ southern Brazil: An estimation of permanent charge and pH-dependent charges. Eur. J. Soil Sci., 57:356-364, 2006.

BRADY, N.C. \& WEIL, R.R. The nature and properties of soils. 13.ed. New Jersey, Upper Saddle River, Prentice Hall, 2002. 960p.

BRINDLEY, G.W. \& BROWN, G. Crystal structures of clays minerals and their X-ray identification. London, Mineralogical Society, 1980. 495p. (Monograph, 5)

CARTER, D.L.; HEIMAN, R. \& GONZALES, C.L. Ethylene glycol monoethyl ether for determining surface area of silicate minerals. Soil Sci., 100:356-360, 1965.

COMISSÃO DE QUÍMICA E FERTILIDADE DO SOLO CQFSRS/SC Recomendações de adubação e calagem para os estados do Rio Grande do Sul e Santa Catarina. 10.ed. Porto Alegre, Sociedade Brasileira de Ciência do Solo, 2004. 400p.

DUCHAUFOUR, P. Abrégé de pédologie: Sol, végétation, environnement. Enseignement des Sciences de la terre. Paris, Masson, 1997. 291p.

DUR, J.C.; ELSASS, F.; CHAPLAIN, V. \& TESSIER, D. The relationship between particle-size distribution by laser granulometry and image analysis by transmission electron microscopy in a soil clay fraction. Eur J. Soil Sci., $55: 265-270,2004$.

DUR, J.C.; WIRIYAKITNATEEKUL, W.; LESTURGEZ, G.; ELSASS, F.; PERNES, M.; HARTMANN, C. \& TESSIER, D. Clay mineral dissolution following intensive cultivation in a tropical sandy soil. In: SYMPOSIUM ON TROPICAL SANDY SOIL, 1., Khon Kaen, 2005. Proceedings. Khon Kaen, 2005. 
ELIMELECH, M. \& RYAN, N.J. The role of mineral colloids in the facilitated transport of contaminants in saturated porous media. In: HUANG, P.M.; BOLLAG, J.M. \& SENESI, N., eds. Interactions between soil particles and microorganisms. Impact on the terrestrial ecosystem. West Sussex, John Wiley Sons, 2002. p.495-548. (IUPAC Series on analytical and physical chemistry of environmental systems, v.8)

EMPRESA BRASILEIRA DE PESQUISA AGROPECUÁRIA EMBRAPA -Manual de métodos de análise de solo. Rio de Janeiro, 1997. 212p.

EMPRESA BRASILEIRA DE PESQUISA AGROPECUÁRIA EMBRAPA. Sistema brasileiro de classificação de solos. Brasília, 2006. 306p.

FAO - World reference base for soil resources - WRB. 2.ed Roma, 2006. 145p. (World Soil Resources Reports, 103)

HOPKINS, D.G. \& FRANZEN, D.W. Argillic horizons in stratified drift: Luverne end Moraine, Eastern North Dakota. Soil Sci. Soc. Am. J., 67:1790-1796, 2003.

KAMPF, N.; AZEVEDO, A.C. \& COSTA, M.I. Estrutura básica de argilominerais 2:1 com hidroxi-Al entrecamadas em Latossolo Bruno do Rio Grande do Sul. R. Bras. Ci. Solo, 19:185-190, 1995.

LAEGDSMAND, M.; MOLDRUP, P. \& DE JONGE, L.W. Modelling of colloid leaching from unsaturated aggregated soil. Eur. J. Soil Sci., 58:692-703, 2007.

LANSON, B. Decomposition of experimental X-ray diffraction patterns (profile fitting): A convenient way to study clay minerals. Clays Clay Miner., 45:132-146, 1997.

LÉGUEDOIS, S.; van OORT, F.; JONGMANS, A.G. \& CHEVALliER, P. Morphology, chemistry and distribution of neoformed mineral species in agricultural land affected by metallurgical point-source pollution. Environ. Poll., 130:135-148, 2004.

MACIEL FILHO, C.L.; SARTORI, P.L.P.; VEIGA, P. \& GASPARETTO, N.V.L. Mapa Geológico da Folha de Camobi. Texto explicativo. Santa Maria, Universidade Federal de Santa Maria, 1988. 10p.

MAFRA, A.L.; SILVA, E.F.; COOPER, M. \& DEMATTÊ, J.L.I. Pedogênese de uma seqüência de solos desenvolvidos de arenito na região de Piracicaba (SP). R. Bras. Ci. Solo, 25:355-369, 2001.

MEHRA, O.P. \& JACKSON, M.L. Iron oxide removal from soils and clays by a dithionite-citrate system buffered with sodium bicarbonate. In: ADA, S., ed. Clays clay mineralogy. Elmsdorf, Pergamon Press, 1960. p.317-342.

MOORE, D.M. \& REYNOLDS, R.C. X-ray diffraction and the identification and analysis of clay minerals. 2.ed. Oxford, Oxford University Press, 1997. 396p.
NOOREN, C.A.M.; van BREEMEN, N.; STOORVOGEL, J.J. \& JONGMANS, A.G. The role of earthworms in the formation of sandy surface soils in a tropical forest in Ivory Coast. Geoderma, 65:135-148, 1995.

OLIVEIRA, L.B.; RIBEIRO, M.R.; FERRAZ, F.B.; FERREIRA, M.G.V.X. \& MERMUT, A.R. Mineralogia, micromorfologia e gênese de solos planossólicos do Sertão do Araripe, estado de Pernambuco. R. Bras. Ci. Solo, 28:665-678, 2004.

PÉDRO, G. Les conditions de formation des constituants secondaires. In: BONNEAU, M. \& SOUCHIER, B., eds. Pédologie: Constituants et propriétés du sol. Paris, Masson, 1979. p.58-71.

PHILLIPS, J.D. Contingency and generalization in pedology, as exemplified by texture-contrast soils. Geoderma, 102:347-370, 2001.

PHILLIPS, J.D. Development of texture contrast soils by combination of bioturbation and translocation. Catena, 70:92-104, 2007.

PHILLIPS, J.D. Geogenesis, pedogenesis, and multiple causality in the formation of texture-contrast soil. Catena, 58:275-295, 2004

ROBERT, M. \& TESSIER, D. Méthode de préparation des argiles des sols pour des études minéralogiques. Ann. Agron., 25:859-882, 1974.

ROUSSEAU, M.; PIETRO, L.D.I.; ANGULO-JARAMILLO, R.; TESSIER, D. \& CABIBEL, B. Preferential transport of soil colloidal particles: Physicochemical effects on particle mobilization. Vadose Zone J., 3:247-261, 2004.

SCHAETZL, R. Lithologic discontinuities in some soils on drumlins theory, detection, and application. Soil Sci., 163:570-590, 1998.

SHOEMAKER, H.E.; MACLEAN, E.O. \& PRATT, P.F. Buffer methods for determining lime requirement of soils with appreciable amounts of extractable aluminum. Soil Sci. Soc. Am. Proc. 25, 274-277, 1961.

SOIL SURVEY STAFF. Soil taxonomy: A basic system of soil classification for making and interpreting soil surveys. 2.ed. Washington, U.S. Department of Agriculture/ Natural Resources Conservations Service, 1999. 871p. (Agriculture Handbook, 436)

van RANST, E. \& CONINCK, F. De evaluation of ferrolysis in soil formation. Eur. J. Soil Sci., 53:513-519, 2002.

WILSON, M.J. The origins and formation of clay minerals in soils: Past, present and future perspectives. Clay Miner., 34:7-25, 1999. 